\title{
A Unified Game-Theoretic Framework for Discrete Power Minimization in Wireless Networks
}

\author{
Wei Zhong \\ PLA University of Science and Technology \\ Email: zhongwei_ch@hotmail.com
}

\author{
Kai Kit Wong \\ University College London \\ Email: kai-kit.wong@ucl.ac.uk
}

\begin{abstract}
This paper proposes a unified noncooperative game for discrete power minimization in wireless networks. We prove that the proposed game, with a carefully chosen payoff function, falls into the framework of potential games where pure strategy Nash equilibrium (NE) exists. The feasibility of the pure strategy $\mathrm{NE}$ is guaranteed with properly designed penalty functions and penalty factors. It is revealed that the optimal solution to the sum discrete power minimization problem constitutes a pure strategy NE of the proposed game under mild conditions. Also, we prove that the pure strategy NE of the proposed game is also the optimal solution to the sum discrete power minimization problem under some particular conditions. An iterative algorithm is then devised to obtain the pure strategy NE. Two examples which can be solved efficiently by using our framework are also provided.
\end{abstract}

\section{INTRODUCTION}

Power control can be used to minimize power consumption and improve energy efficiency in wireless networks and has received much attention in research. The study of power control can be generally divided into two categories: (1) power control with continuous strategies [1]-[7] and (2) power control with discrete strategies [8]-[13].

While power is naturally continuous, in practice, there are only a number of quantized values to operate at [8]. The study of discrete power control is therefore of practical relevance and significance. However, the existing literature in discrete power control is limited to the application in cellular networks under some conditions [8]-[10], [14]-[16]. For examples, the framework in [14] needs that the interference function is standard, [10] only consider several typical constraints (e.g., average power constraints), the game model in [15] needs that the utility function of the game player should be supermodular or submodular. Discrete power control for more general wireless networks, e.g., with non standard problems, such as cognitive radio networks, D2D communication systems, and so on, is much less understood.

Motivated by [2], [11]-[13], this paper presents a unified game-theoretic framework for constrained discrete power minimization problems. Our main contribution is the design of the payoff function and the penalty function such that the feasibility of the Nash equilibrium (NE) ensures satisfaction of the constraints. Based on the our specially designed functions, the application scenarios of the framework in this paper are much wider than the previous works. Furthermore, we prove that the proposed game is a potential game and give the closed form expression of the potential function. We also prove that the maximizer of the potential function is the optimal solution to the discrete sum power minimization problem under mild conditions. An iterative power updating algorithm is proposed to achieve the feasible pure strategy NE. Furthermore, two examples are given to assess our proposed game-theoretic framework.

Note that, [2] and [16] also apply potential game to the power control problems in wireless networks. However, the theoretic results in [2] mainly lies in the case of continuous strategies and only a standard power minimization problem with relative simple constraints is preliminary studied. [16] only consider a special linear utility model when potential game is applied and no coupled constraints are taken into account.

\section{Discrete Power Minimization Game}

\section{A. Game-Theoretic Formulation}

Consider a wireless network of $n$ users where the transmit power level of user $i \in \mathcal{N}=\{1,2, \ldots, n\}$, denoted by $p_{i}$, is chosen from a finite set $\mathcal{P}_{i}=\left\{P_{i 1}, \ldots, P_{i M_{i}}\right\}$ where $P_{i j}$ denotes the $j$ th power level of user $i$, and $M_{i}$ is the cardinality of $\mathcal{P}_{i}$. To minimize power consumption, each user aims to minimize its transmit power while the quality-of-service (QoS) requirements or some other constraints are satisfied. Formally, the discrete power minimization problem can be written as

$$
\begin{aligned}
\min _{p_{i} \in \mathcal{P}_{i}} & p_{i} \\
\text { s.t. } & \left\{\begin{array}{l}
g_{k}\left(p_{1}, \ldots, p_{n}\right) \geq 0, \text { for } k \in \mathcal{K}, \\
\mathrm{NZP},
\end{array}\right.
\end{aligned}
$$

where $g_{k}\left(p_{1}, \ldots, p_{n}\right) \geq 0$ denotes any given constraint in the wireless network, and $\mathcal{K}=\{1,2, \ldots, K\}$. These constraints may be coupled in wireless networks [2], [10], [14]. Additionally, NZP means that $p_{i}$ cannot be zero unless some of the constraints cannot be met if $p_{i}>0$ for a given power strategy tuple of the other users. Hence, users are encouraged to be active rather than shut down if a feasible power strategy $p_{i}>0$ exists. From the game-theoretic viewpoint, (1) can be formulated as a non-cooperative game

$$
\mathcal{G}_{1}=\left[\mathcal{N},\left\{\mathcal{S}_{i}\right\}_{i \in \mathcal{N}},\left\{p_{i}\right\}_{i \in \mathcal{N}}\right]
$$

where $\left\{\mathcal{S}_{i}\right\}_{i \in \mathcal{N}}$ denotes the set of feasible power (i.e., pure strategy) profiles of user $i$ and $p_{i}$ is the payoff of user $i$. 
However, $\mathcal{G}_{1}$ is impractical because it is usually difficult for the users to know the set of all feasible power profiles, i.e., $\left\{\mathcal{S}_{i}\right\}_{i \in \mathcal{N}}$ in advance. To overcome this problem, we propose a new game $\mathcal{G}_{2}$ by introducing the penalty function so that

$$
\mathcal{G}_{2}=\left[\mathcal{N},\left\{\mathcal{P}_{i}\right\}_{i \in \mathcal{N}},\left\{u_{i}\right\}_{i \in \mathcal{N}}\right],
$$

where $u_{i}$ is defined as

$$
u_{i}\left(p_{i}, \boldsymbol{p}_{-i}\right) \triangleq \begin{cases}\alpha-p_{i}+f\left(p_{i}, \boldsymbol{p}_{-i}\right), & \text { if } p_{i}>0 \\ \alpha-\frac{\beta}{2 n}+f\left(0, \boldsymbol{p}_{-i}\right), & \text { if } p_{i}=0\end{cases}
$$

in which $\alpha$ is a positive scalar whose value will be given later, $\boldsymbol{p}_{-i}=\left(\left\{p_{j}\right\}_{j \in \mathcal{N} \backslash i}\right)$ denotes the powers of all users excluding the $i$-th user, $f\left(p_{i}, \boldsymbol{p}_{-i}\right)$ denotes the penalty function [12] of the combinational constraints which is defined as

( $-\beta+n_{0} \alpha, \quad$ if $\exists i \in \mathcal{N}$ and $\exists k \in \mathcal{K}$, s.t. $p_{i}>0$ $f\left(p_{i}, \boldsymbol{p}_{-i}\right) \triangleq\left\{\begin{array}{cl} & \text { and the } k \text {-th constraint is violated } \\ 0, & \text { otherwise }\end{array}\right.$

where the penalty factor $\beta$ is a positive scalar which can be pre-stored at all nodes in the network and $n_{0}$ is the number of users whose transmit power is zero. The penalty function $f$ reflects the constraints in the wireless network.

Formally, the discrete noncooperative power minimization game $\mathcal{G}_{2}$ can be expressed as

$$
\max _{p_{i} \in \mathcal{P}_{i}} u_{i}\left(p_{i}, \boldsymbol{p}_{-i}\right) \text {. }
$$

Note that, since $\mathcal{G}_{2}$ does not need to know the feasible power profile set and each user does not need to know the powers of other users', $\mathcal{G}_{2}$ is much more practical than $\mathcal{G}_{1}$. Moreover, it will be shown later that the feasibility of the solution of $\mathcal{G}_{2}$ can be guaranteed by a properly designed value of $\beta$ even in the case of not knowing the infeasible strategy profile.

\section{B. Theoretical Analysis and Results}

In this subsection, we give theoretic analysis of the proposed game. To begin with, we define

$$
\psi\left(p_{1}, \ldots, p_{n}\right) \triangleq \sum_{i \in \mathcal{N}}\left(\alpha-p_{i}\right)+f\left(p_{1}, \ldots, p_{n}\right)-\frac{\beta n_{0}}{2 n}
$$

Theorem 1: We have the following main results for $\mathcal{G}_{2}$ :

(i) $\mathcal{G}_{2}$ is a potential game with potential function $\psi$;

(ii) The maximizer of $\psi$ is a pure strategy $\mathrm{NE}$ of $\mathcal{G}_{2}$ and $\mathcal{G}_{2}$ possesses at least one pure strategy NE;

(iii) Given that $\beta=2 n \alpha$ and $\alpha>\sum_{i \in \mathcal{N}} \bar{p}_{i}$ (where $\bar{p}_{i} \triangleq$ $P_{i M_{i}}$ ), the pure strategy NE of $\mathcal{G}_{2}$ must be feasible.

Proof: We first present the proof of (i). It is easy to see that $\forall p_{i}, p_{i}^{\prime} \in \mathcal{P}_{i}$,

$$
\begin{aligned}
& u_{i}\left(p_{i}, \boldsymbol{p}_{-i}\right)-u_{i}\left(p_{i}^{\prime}, \boldsymbol{p}_{-i}\right) \\
& =\left\{\begin{array}{cc}
p_{i}^{\prime}-p_{i}+f\left(p_{i}, \boldsymbol{p}_{-i}\right)-f\left(p_{i}^{\prime}, \boldsymbol{p}_{-i}\right), & \text { if } p_{i}>0, p_{i}^{\prime}>0, \\
\frac{\beta}{2 n}-p_{i}+f\left(p_{i}, \boldsymbol{p}_{-i}\right)-f\left(0, \boldsymbol{p}_{-i}\right), & \text { if } p_{i}>0, p_{i}^{\prime}=0, \\
p_{i}^{\prime}-\frac{\beta}{2 n}-f\left(p_{i}^{\prime}, \boldsymbol{p}_{-i}\right)+f\left(0, \boldsymbol{p}_{-i}\right), & \text { if } p_{i}=0, p_{i}^{\prime}>0,
\end{array}\right. \\
& =\psi\left(p_{i}, \boldsymbol{p}_{-i}\right)-\psi\left(p_{i}^{\prime}, \boldsymbol{p}_{-i}\right) .
\end{aligned}
$$

Thus, $\mathcal{G}_{2}$ satisfies the definition of a potential game in [18] and is a potential game where $\psi$ is the potential function.
Next is the proof of (ii). Since $\mathcal{G}_{2}$ is a potential game, then from [18, Lemma 2.1], we can obtain that the maximizer of the potential function $\psi$ is a pure strategy $\mathrm{NE}$ of $\mathcal{G}_{2}$.

Now, we prove (iii) by the method of contradiction. Suppose that $\forall i \in \mathcal{N}$ and $\forall k \in \mathcal{K},\left(p_{i}, \boldsymbol{p}_{-i}\right)$ is a pure strategy NE of $\mathcal{G}_{2}$ but violates one of the constraints. Then $\left(p_{i}, \boldsymbol{p}_{-i}\right) \neq\left(\{0\}_{i \in \mathcal{N}}\right)$ and $u_{i}\left(p_{i}, \boldsymbol{p}_{-i}\right)=\alpha-p_{i}-\beta+n_{0} \alpha<0$. Since

$$
u_{i}\left(0, \boldsymbol{p}_{-i}\right)=\alpha-\frac{\beta}{2 n}+f\left(0, \boldsymbol{p}_{-i}\right),
$$

we have

$u_{i}\left(p_{i}, \boldsymbol{p}_{-i}\right)-u_{i}\left(0, \boldsymbol{p}_{-i}\right)=\frac{\beta}{2 n}-p_{i}-\beta-f\left(0, \boldsymbol{p}_{-i}\right)=-p_{i}<0$

Thus, (10) contradicts the assumption that $\left(p_{i}, \boldsymbol{p}_{-i}\right)$ is a pure dstrategy NE of $\mathcal{G}_{2}$ according to the definition of pure strategy $\mathrm{NE}$ in [17]. In other words, if $\left(p_{i}, \boldsymbol{p}_{-i}\right)$ violates a constraint, it is not a pure strategy NE of $\mathcal{G}_{2}$. Or, if the conditions of the theorem hold, the pure strategy NE of $\mathcal{G}_{2}$ must be feasible.

From Theorem 1, we know that the feasibility and existence of the pure strategy NE of $\mathcal{G}_{2}$ is guaranteed by a properly designed penalty factor. However, it is unclear if such properties hold in $\mathcal{G}_{1}$. The following corollary studies this issue.

Corollary 1: Suppose that $\beta=2 n \alpha$ and $\alpha>\sum_{i \in \mathcal{N}} \bar{p}_{i}$, a power profile is the pure strategy $\mathrm{NE}$ of $\mathcal{G}_{1}$ if and only if it is the pure strategy NE of $\mathcal{G}_{2}$.

Proof: We first prove the "only if" part. Assume that $\left(p_{1}^{*}, \ldots, p_{n}^{*}\right)$ is the pure strategy $\mathrm{NE}$ of $\mathcal{G}_{1}$. Then from the definition of the pure strategy NE in [17], it is known that $\left(p_{1}^{*}, \ldots, p_{n}^{*}\right)$ must be feasible, and

$$
p_{i}^{*} \leq p_{i}, p_{i}^{*} \in \mathcal{S}_{i}, \forall p_{i} \in \mathcal{S}_{i}, p_{i} \neq p_{i}^{*}, \forall i \in \mathcal{N} .
$$

Since $\beta=2 n \alpha$ and $\alpha>\sum_{i \in \mathcal{N}} \bar{p}_{i}$, we have

$$
\alpha-p_{i}^{*} \geq \alpha-p_{i}, p_{i}^{*} \in \mathcal{S}_{i}, \forall p_{i} \in \mathcal{S}_{i}, p_{i} \neq p_{i}^{*}, \forall i \in \mathcal{N} .
$$

Obviously, $\left(p_{1}^{*}, \ldots, p_{n}^{*}\right)$ is the pure strategy NE of $\mathcal{G}_{2}$.

Now, we proceed to prove the "if" part. Suppose that $\left(p_{1}^{*}, \ldots, p_{n}^{*}\right)$ is the pure strategy NE of $\mathcal{G}_{2}$. Then from Theorem 1 , we have that $\left(p_{1}^{*}, \ldots, p_{n}^{*}\right)$ must be feasible. Therefore,

$$
\alpha-p_{i}^{*}>\alpha-p_{i}, p_{i}^{*} \in \mathcal{S}_{i}, \forall p_{i} \in \mathcal{S}_{i}, p_{i} \neq p_{i}^{*}, \forall i \in \mathcal{N} .
$$

Since $\alpha>\sum_{i \in \mathcal{N}} \bar{p}_{i}$, we then have

$$
p_{i}^{*}<p_{i}, p_{i}^{*} \in \mathcal{S}_{i}, \forall p_{i} \in \mathcal{S}_{i}, p_{i} \neq p_{i}^{*}, \forall i \in \mathcal{N} .
$$

As a result, $\left(p_{1}^{*}, \ldots, p_{n}^{*}\right)$ is also the pure strategy NE of $\mathcal{G}_{1}$. Hence the result of this corollary is proved.

From Theorem 1 and Corollary 1, it can be understood that the existence and feasibility of the pure strategy NE of $\mathcal{G}_{1}$ are guaranteed by a penalty factor which is larger than an easily obtained threshold.

\section{Algorithm}

Here, we propose a discrete power minimization algorithm based on $\mathcal{G}_{2}$. The algorithm is referred to as an iterative power update (IPU) algorithm based on best response dynamic. 


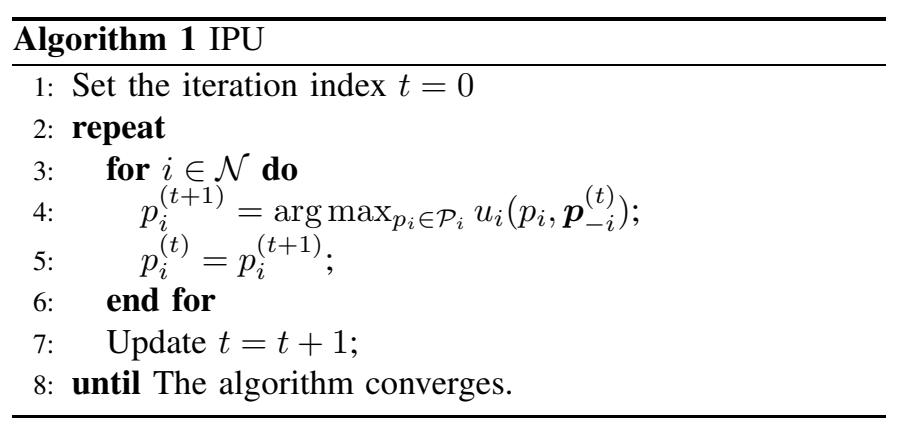

Theorem 2: Suppose that $\beta=2 n \alpha$ and $\alpha>\sum_{i \in \mathcal{N}} \bar{p}_{i}$, IPU converges to a feasible pure strategy NE of $\mathcal{G}_{1}$ and $\mathcal{G}_{2}$ in a finite number of steps from any initial points.

Proof: In IPU, once a user changes its power level, we regard it as a strategy change event. Let $t^{j}$ denote the time instant when the $j$-th strategy change event happens.

Then if the conditions of this theorem hold, once active user $i$ changes its power level at time instant $t^{j}$, we have

$$
u_{i}\left(p_{i}^{\left(t^{j}\right)}, \boldsymbol{p}_{-i}^{\left(t^{j}\right)}\right)-u_{i}\left(p_{i}^{\left(t^{j}-1\right)}, \boldsymbol{p}_{-i}^{\left(t^{j}\right)}\right)>0 .
$$

Since $\mathcal{G}_{2}$ is a potential game with potential function $\psi$, then

$$
\begin{aligned}
\psi & \left(p_{i}^{\left(t^{j}\right)}, \boldsymbol{p}_{-i}^{\left(t^{j}\right)}\right)-\psi\left(p_{i}^{\left(t^{j}-1\right)}, \boldsymbol{p}_{-i}^{\left(t^{j}\right)}\right) \\
& =u_{i}\left(p_{i}^{\left(t^{j}\right)}, \boldsymbol{p}_{-i}^{\left(t^{j}\right)}\right)-u_{i}\left(p_{i}^{\left(t^{j}-1\right)}, \boldsymbol{p}_{-i}^{\left(t^{j}\right)}\right)>0 .
\end{aligned}
$$

Therefore, the payoff of each user and $\psi$ are strictly increasing. Since the power levels of each user are finite and the maximum value of $\psi$ is usually finite, i.e., $\psi<\infty$, IPU must converge in a finite number of steps.

When IPU converges to a power profile $\left(p_{1}^{*}, \ldots, p_{n}^{*}\right)$, for an arbitrary user $i$ and an alternative pure strategy $p_{i}$, we have

$$
u_{i}\left(p_{i}^{*}, \boldsymbol{p}_{-i}^{*}\right)-u_{i}\left(p_{i}, \boldsymbol{p}_{-i}^{*}\right)>0 .
$$

According to [17], the power profile $\left(p_{1}^{*}, \ldots, p_{n}^{*}\right)$ is a pure strategy NE of $\mathcal{G}_{2}$. From Theorem 1 and Corollary 1 , we have that $\left(p_{1}^{*}, \ldots, p_{n}^{*}\right)$ must be feasible and is also the pure strategy NE of $\mathcal{G}_{1}$ if $\beta 2 n \alpha$ and $\alpha>\sum_{i \in \mathcal{N}} \bar{p}_{i}$.

\section{OptimALITy}

\section{A. Discrete Sum Power Minimization}

In the $n$-user wireless network, the discrete sum power minimization problem can be formulated as

$$
\begin{aligned}
\min _{\left(p_{1}, \ldots, p_{n}\right) \in \mathcal{P}} & \sum_{i \in \mathcal{N}} p_{i} \\
\text { s.t. } & \left\{\begin{array}{l}
g_{k}\left(p_{1}, \ldots, p_{n}\right) \geq 0, \text { for } k \in \mathcal{K}, \\
\mathrm{NZPCO},
\end{array}\right.
\end{aligned}
$$

where $\mathcal{P}=\mathcal{P}_{1} \times \mathcal{P}_{2} \times \cdots \times \mathcal{P}_{n}$ is the set of all possible discrete power strategy profiles, and NZPCO means "non-zero power constraint", i.e., for any user $i, p_{i}$ cannot be zero unless any of the constraints cannot be met if $p_{i}>0$ no matter what other users' powers are.
To establish the relationship between (18) and our designed game, we define the discrete optimization problem:

$$
\max _{\left(p_{1}, \ldots, p_{n}\right) \in \mathcal{P}} \psi\left(p_{1}, \ldots, p_{n}\right) .
$$

Lemma 1: Given that we have $\beta=2 n \alpha$ and $\alpha>\sum_{i \in \mathcal{N}} \bar{p}_{i}$, $\left(p_{1}^{\text {opt }}, \ldots, p_{n}^{\text {opt }}\right)$ is the optimal solution to problem (18) if and only if it is the optimal solution to problem (19).

Proof: First, we have the proof of the "if" part. Suppose that $\left(p_{1}^{\text {opt }}, \ldots, p_{n}^{\text {opt }}\right)$ is the optimal solution to (19). Then

$$
\begin{aligned}
& \psi\left(p_{1}^{\mathrm{opt}}, \ldots, p_{n}^{\mathrm{opt}}\right) \geq \psi\left(p_{1}, \ldots, p_{n}\right) \\
& \quad \forall\left(p_{1}, \ldots, p_{n}\right) \in \mathcal{P},\left(p_{1}, \ldots, p_{n}\right) \neq\left(p_{1}^{\mathrm{opt}}, \ldots, p_{n}^{\mathrm{opt}}\right) .
\end{aligned}
$$

That is,

$$
\begin{gathered}
\sum_{i \in \mathcal{N}}\left(\alpha-p_{i}^{\mathrm{opt}}\right)+f\left(p_{1}^{\mathrm{opt}}, \ldots, p_{n}^{\mathrm{opt}}\right)-\frac{n_{0}^{\mathrm{opt}} \beta}{2 n} \\
\geq \sum_{i \in \mathcal{N}}\left(\alpha-p_{i}\right)+f\left(p_{1}, \ldots, p_{n}\right)-\frac{n_{0} \beta}{2 n} \\
\forall\left(p_{1}, \ldots, p_{n}\right) \in \mathcal{P},\left(p_{1}, \ldots, p_{n}\right) \neq\left(p_{1}^{\mathrm{opt}}, \ldots, p_{n}^{\mathrm{opt}}\right) .
\end{gathered}
$$

Assuming that $\left(p_{1}^{\text {opt }}, \ldots, p_{n}^{\text {opt }}\right)$ violates at least one of the constraints, then we have

$$
f\left(p_{1}^{\mathrm{opt}}, \ldots, p_{n}^{\mathrm{opt}}\right)=-\beta+n_{0}^{\mathrm{opt}} \alpha<0 .
$$

If the conditions of this lemma hold, then

$$
\begin{aligned}
\psi\left(p_{1}^{\mathrm{opt}}, \ldots, p_{n}^{\mathrm{opt}}\right) & =\sum_{i \in \mathcal{N}}\left(\alpha-p_{i}^{\mathrm{opt}}\right)-\beta+n_{0}^{\mathrm{opt}} \alpha-\frac{n_{0}^{\mathrm{opt}} \beta}{2 n} \\
& <\psi(0, \ldots, 0)=\sum_{i \in \mathcal{N}} \alpha-\frac{\beta}{2} .
\end{aligned}
$$

Therefore, (23) contradicts (20) and that $\left(p_{1}^{\mathrm{opt}}, \ldots, p_{n}^{\mathrm{opt}}\right)$ must be feasible and $f\left(p_{1}^{\text {opt }}, \ldots, p_{n}^{\text {opt }}\right)=0$.

Suppose that $\left(p_{1}^{\prime}, \ldots, p_{n}^{\prime}\right) \in \mathcal{P}$ is the optimal solution to (18) and $\left(p_{1}^{\prime}, \ldots, p_{n}^{\prime}\right) \neq\left(p_{1}^{\text {opt }}, \ldots, p_{n}^{\text {opt }}\right)$. Then from (21), we have $f\left(p_{1}^{\prime}, \ldots, p_{n}^{\prime}\right)=0$ and $\sum_{i \in \mathcal{N}} p_{i}^{\prime}+\frac{n_{0}^{\prime} \beta}{2 n} \leq \sum_{i \in \mathcal{N}} p_{i}^{\text {opt }}+$ $\frac{n_{0}^{\text {opt } \beta}}{2 n}$, which can be re-expressed into

$$
\sum_{i \in \mathcal{N}}\left(\alpha-p_{i}^{\prime}\right)-\frac{n_{0}^{\prime} \beta}{2 n} \geq \sum_{i \in \mathcal{N}}\left(\alpha-p_{i}^{\mathrm{opt}}\right)-\frac{n_{0}^{\mathrm{opt}} \beta}{2 n} .
$$

Therefore, (24) contradicts (20) and (21), which implies that $\left(p_{1}^{\prime}, \ldots, p_{n}^{\prime}\right)=\left(p_{1}^{\mathrm{opt}}, \ldots, p_{n}^{\mathrm{opt}}\right)$. That is, if $\left(p_{1}^{\mathrm{opt}}, \ldots, p_{n}^{\mathrm{opt}}\right)$ is the optimal solution to (19), it is also the optimal solution to (18). Using the similar procedure, we can easily prove the "only if" part to complete the proof.

\section{B. Relationship between the Optimal Solution and the NE}

In this subsection, we study the relation between the optimal solution to the sum discrete power minimization problem (19) and the pure strategy NE of the non-cooperative power minimization game. First, we give the following theorem.

Theorem 3: Given that $\beta=2 n \alpha$ and $\alpha>\sum_{i \in \mathcal{N}} \bar{p}_{i}$, the optimal solution to (18) constitutes a feasible pure strategy NE of $\mathcal{G}_{1}$ and $\mathcal{G}_{2}$. 
Proof: Suppose that $\left(p_{1}^{\mathrm{opt}}, \ldots, p_{n}^{\mathrm{opt}}\right)$ is the optimal solution to (18). From Lemma 1 , we know that $\left(p_{1}^{\mathrm{opt}}, \ldots, p_{n}^{\mathrm{opt}}\right)$ is the optimal solution to (19). Since we have proved in Theorem 1 that the maximizer of $\psi$ is a feasible pure strategy NE of $\mathcal{G}_{2}$ if $\beta=2 n \alpha$ and $\alpha>\sum_{i \in \mathcal{N}} \bar{p}_{i}$. Thus, $\left(p_{1}^{\text {opt }}, \ldots, p_{n}^{\text {opt }}\right)$ is a feasible pure strategy $\mathrm{NE}$ of $\mathcal{G}_{2}$ when the conditions of this theorem holds. Next, from Corollary $1,\left(p_{1}^{\mathrm{opt}}, \ldots, p_{n}^{\mathrm{opt}}\right)$ is also a feasible pure strategy NE of $\mathcal{G}_{1}$.

Theorem 3 reveals the relationship between the NE of our proposed game the optimal solution to (18). It shows that the set of the optimal solutions to (18) is a subset of the pure strategy NE of our proposed game in general. From Theorem 3 , we can further have the following corollary.

Corollary 2: Given $\beta=2 n \alpha$ and $\alpha>\sum_{i \in \mathcal{N}} \bar{p}_{i}$, we have:

(i) If $\mathcal{G}_{2}$ possesses a unique pure strategy $\mathrm{NE}$, this unique pure strategy $\mathrm{NE}$ is the optimal solution to (18);

(ii) If $\forall\left(p_{1}, \ldots, p_{n}\right) \in \mathcal{P},\left(p_{1}, \ldots, p_{n}\right) \neq(0, \ldots, 0)$ cannot satisfy the stated constraints $g_{1}, \ldots, g_{K}$, then the unique pure strategy $\mathrm{NE}$ of $\mathcal{G}_{2}$ is $(0, \ldots, 0)$ and is also the optimal solution to (18).

Proof: From Theorem 1 and Theorem 3, we immediately reach the result in (i). Then we proceed to prove part (ii).

Suppose that we have an arbitrary power strategy profile $\left(p_{1}, \cdots, p_{n}\right) \in \mathcal{P}$ and that $\left(p_{1}, \ldots, p_{n}\right) \neq(0, \ldots, 0)$ cannot satisfy the stated constraints $g_{1}, \ldots, g_{K}$ which is also a pure strategy NE of $\mathcal{G}_{2}$. If the condition of (i) holds, we have

$$
\begin{aligned}
& \psi(0, \ldots, 0)-\psi\left(p_{1}, \ldots, p_{n}\right) \\
& =-\frac{\beta}{2}+\sum_{i \in \mathcal{N}} p_{i}+\beta-n_{0} \alpha+\frac{n_{0} \beta}{2 n}=\sum_{i \in \mathcal{N}} p_{i}+n \alpha>0,
\end{aligned}
$$

which contradicts the assumption that $\left(p_{1}, \ldots, p_{n}\right)$ is a pure strategy NE of $\mathcal{G}_{2}$. Thus, $\left(p_{1}, \ldots, p_{n}\right)$ cannot be the pure strategy NE of $\mathcal{G}_{2}$. Since (25) always holds $\forall\left(p_{1}, \ldots, p_{n}\right) \in \mathcal{P}$ and $\left(p_{1}, \ldots, p_{n}\right) \neq(0, \ldots, 0),(0, \ldots, 0)$ is the unique pure strategy NE of $\mathcal{G}_{2}$ and is also the optimal solution to (19). From Lemma 1 and Theorem 3, we know that $(0, \ldots, 0)$ is also the optimal solution to (18).

\section{NUMERICAL EXAMPLES}

In this section, we provide two examples to evaluate the performance of our proposed scheme.

\section{A. Cognitive Radio Systems}

In this subsection, we assumed a network of $n$ cognitive users coexisting with $L$ primary users sharing the same timefrequency resource. One user corresponds to a transmitter-toreceiver link. To protect the primary users, the interference power constraints at the primary receivers are in place. For each primary user $l \in \mathcal{L}=\{1,2, \ldots, L\}$, we require

$$
P_{l}^{M}-\sum_{i=1}^{n} p_{i}\left|H_{i l}\right|^{2} \geq 0,
$$

where $P_{l}^{M}$ denotes the interference power constraint at the receiver of primary user $l, p_{i} \in \mathcal{P}_{i}$ denotes the transmit power of cognitive user $i$, and $H_{i l}$ denotes the channel gain from cognitive user $i$ to the receiver of primary user $l$.

To ensure the QoS of each cognitive user, it is also assumed that the signal-to-interference-and-noise ratio (SINR) of cognitive user $i$, denoted as $\gamma_{i}$, should be greater than a preset threshold $\gamma_{i}^{M}$. For each cognitive user $i \in \mathcal{N}$, we have

$$
\gamma_{i}-\gamma_{i}^{M} \geq 0
$$

where

$$
\gamma_{i}=\frac{p_{i}\left|h_{i i}\right|^{2}}{\sum_{\substack{j \neq i \\ j=1}}^{n} p_{j}\left|h_{j i}\right|^{2}+\sum_{l=1}^{L} q_{l}\left|g_{l i}\right|^{2}+\sigma^{2}},
$$

and $h_{j i}$ denotes the channel gain from cognitive transmitter $j$ to cognitive receiver $i, q_{l}$ denotes the transmit power of primary user $l, g_{l i}$ denotes the channel gain from primary transmitter $l$ to secondary receiver $i$.

In the simulations, we have assumed that $L=2, \sigma^{2}=$ $5 \times 10^{-10}$ Watts, $\mathcal{P}_{i}=\{0,0.005,0.05,0.5\}$ Watts, $\gamma_{i}^{M}=$ $3 \mathrm{~dB}, \forall i \in \mathcal{N}$, and $q_{l}=0.05$ Watts, $P_{l}^{M}=0.01$ Watts, $\forall l \in$ $\mathcal{L}$.

Figs. 1 illustrates the convergence performance of IPU with 4 cognitive users and 2 primary users. Results show that IPU converges and the speed of convergence is very fast. This result also means that the NE of our proposed game always exists.

Furthermore, we compare the sum power results obtained by exhaustive search (ES) and IPU with 1000 channel realizations in a 4 cognitive users and 2 primary users system and find that the percentage of that the result of IPU is the same as that of ES is $95.1 \%$. This result means that IPU can directly find the optimal solution to problem (18) with very high probability and also verify the correctness that the optimal solution to problem (18) is also an NE of our proposed game.

\section{B. D2D Communication Systems}

In this subsection, we considered a D2D underlay communications network as an example to demonstrate the effectiveness of the proposed framework. In particular, we assumed a network of $n$ D2D users coexisting with $L$ cellular users sharing the same time-frequency resource. One user corresponds to a transmitter-to-receiver link. To protect the communication of each cellular user $l \in \mathcal{L}=\{1,2, \ldots, L\}$, we require

$$
\gamma_{l}-\gamma_{l}^{M} \geq 0
$$

where

$$
\gamma_{l}=\frac{q_{l}\left|G_{l l}\right|^{2}}{\sum_{j=1}^{n} p_{j}\left|H_{j l}\right|^{2}+\sum_{\substack{j \neq l \\ j=1}}^{L} q_{j}\left|G_{j l}\right|^{2}+\sigma_{l}^{2}}
$$

$\gamma_{l}^{M}$ denotes the minimum signal-to-interference-and-noise ratio (SINR) constraint at the receiver of cellular user $l, q_{l} \in \mathcal{Q}_{l}$ denotes the transmit power of cellular user $l, p_{i} \in \mathcal{P}_{i}$ denotes the transmit power of D2D user $i, G_{j l}$ denotes the channel gain from the transmitter of cellular user $j$ to the receiver of cellular user $l$, and $H_{i l}$ denotes the channel gain from D2D user $i$ to the receiver of cellular user $l$. 




Fig. 1. Convergence of IPU with 4 cognitive users and 2 primary users.

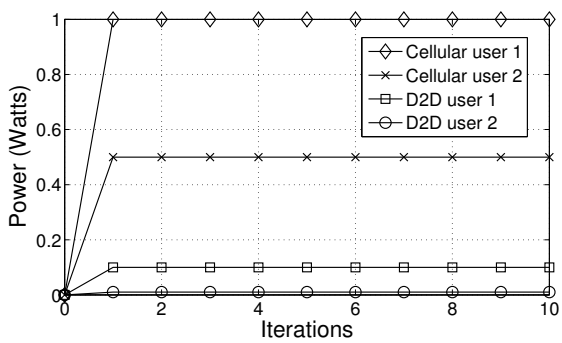

Fig. 2. Convergence of IPU with 2 cellular users and 2 D2D users.

To ensure the QoS of each D2D user, it is also assumed that the SINR of D2D user $i$, denoted as $\gamma_{i}$, should be greater than a preset threshold $\gamma_{i}^{M}$. For each D2D user $i \in \mathcal{N}$, we have

$$
\gamma_{i}-\gamma_{i}^{M} \geq 0
$$

where

$$
\gamma_{i}=\frac{p_{i}\left|h_{i i}\right|^{2}}{\sum_{\substack{j \neq i \\ j=1}}^{n} p_{j}\left|h_{j i}\right|^{2}+\sum_{l=1}^{L} q_{l}\left|g_{l i}\right|^{2}+\sigma_{i}^{2}},
$$

and $h_{j i}$ denotes the channel gain from the transmitter of D2D user $j$ to the receiver of D2D user $i, g_{l i}$ denotes the channel gain from cellular transmitter $l$ to the receiver of D2D user $i$.

In the simulations, we assume that $\sigma_{l}^{2}=\sigma_{i}^{2}=$ $10^{-13}$ Watts, $\mathcal{Q}_{l}=\{0.001,0.005,0.05,0.5,1\}$ Watts, $\mathcal{P}_{i}=$ $\{0,0.001,0.01,0.1,0.5\}$ Watts, $\gamma_{l}^{M}=10 \mathrm{~dB}, \gamma_{i}^{M}=5 \mathrm{~dB}$, $\forall i \in \mathcal{N}, \forall l \in \mathcal{L}$. Furthermore, the initial power strategy of each user in IPU is the minimum power level of it.

Figs. 2 and 3 illustrate the convergence performance of IPU with different numbers of cellular users and D2D users. Again, the results show that IPU always converges very fast. In addition, we compare the sum power results obtained by exhaustive search (ES) and IPU with 1000 channel realizations in a 2 cellular users and 2 D2D users system and find that the percentage of that the result of IPU is the same as that of ES is $85.8 \%$. This result again shows that IPU can directly find the optimal solution to problem (18) with high probability.

\section{CONCLUSION}

This paper proposed a unified game-theoretic framework to address the discrete power minimization problem in wireless networks. By designing the penalty-based payoff function, we have given the potential function and proved that our proposed

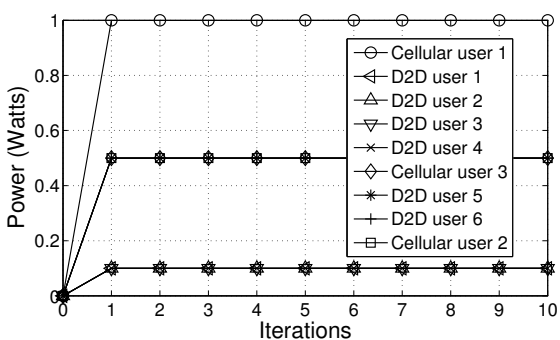

Fig. 3. Convergence of IPU with 3 cellular users and 6 D2D users.

game is a potential game in which pure strategy NE must exist and the maximizer of the potential function coincides with the optimal solution to the sum discrete power minimization problem under mild conditions. The IPU algorithm was proposed to obtain the pure strategy NE. Numerical results for cognitive radio networks and D2D communication systems as examples were provided to assess the approach.

\section{ACKNOWLEDGMENT}

This work was supported by Jiangsu Province Natural Science Foundation under Grant BK20160080.

\section{REFERENCES}

[1] C. Saraydar, N. B. Mandayam, and D. J. Goodman, "Efficient power control via pricing in wireless data networks," IEEE Trans. Commun., vol. 50, pp. 291-303, Feb. 2002.

[2] G. Scutari, S. Barbarossa, and D.P. Palomar, "Potential games: A framework for vector power control problems with coupled constraints," in Proc. IEEE ICASSP, Toulouse, France, May 2006, pp. 14-19.

[3] Z. Wang, L. Jiang, and C. He, "Optimal price-based power control algorithm in cognitive radio networks," IEEE Trans. Wireless Commun., vol. 13 , no. 11 , pp. 5909-5920, Nov. 2014.

[4] M. Monemi, M. Rasti, and E. Hossain, "On joint power and admission control in underlay cellular cognitive radio networks," IEEE Trans. Wireless Commun., vol. 14, no. 1, pp. 265-278, Jan. 2015.

[5] Q. Wang, W. Wang, S. Jin, H. Zhu, and N. T. Zhang, "Quality-optimized joint source selection and power control for wireless multimedia D2D communication using stackelberg game," IEEE Trans. Vech. Tech., vol. 64 , no. 8 , pp. $3755-3769$, Aug. 2015.

[6] N. Lee, X. Lin, J. G. Andrews, and R. W. Heath, Jr., "Power control for D2D underlaid cellular networks: modeling, algorithms, and analysis," IEEE J. Sel. Areas in Commun., vol. 33, no. 1, pp. 1-13, Jan. 2015.

[7] W. Wang, F. Zhang, and V. K. N. Lau,"Dynamic power control for delay-aware Device-to-Device communications," IEEE J. Sel. Areas in Commun., vol. 33, no. 1, pp. 14-27, Jan. 2015.

[8] M. Andersin, Z. Rosberg, and J. Zander, "Distributed discrete power control in cellular PCS," Wireless Per. Commun., vol. 6, no. 3, pp. 211231, 1998.

[9] Y. Xing, and R. Chandramouli, "Stochastic learning solution for distributed discrete power control game in wireless data networks," IEEE/ACM Trans. Net., vol. 16, no. 4, pp. 932-944, Aug. 2008.

[10] E. Altman, K. Avrachenkov, I. Menache, G. Miller, B. J. Prabhu, and A. Shwartz, "Dynamic discrete power control in cellular networks," IEEE Trans. Auto. Control, vol. 54, no. 10, pp. 2328-2340, Oct. 2009.

[11] W. Zhong and J. Wang, "Energy efficient spectrum sharing strategy selection in cognitive MIMO interference channels," IEEE Trans. Sig. Process., vol. 61, no. 14, pp. 3705-3717, Jul. 2013.

[12] W. Zhong, G. Chen, S. Jin, and K. K. Wong, "Relay selection and discrete power control for cognitive relay networks via potential game," IEEE Trans. Sig. Process., vol. 62, no. 20, pp. 5411-5424, 2014

[13] W. Zhong, Y. Fang, S. Jin, K. K. Wong, S. Zhong, and Z. Qian, "Joint resource allocation for Device-to-Device communications underlaying uplink MIMO cellular networks," IEEE J. Sel. Areas in Commun., vol. 33 , no. 1 , pp. 41-54, 2015. 
[14] R. D. Yates, "A framework for uplink power control in cellular radio systems," IEEE J. Sel. Areas in Commun., vol. 13, no. 7, pp. 1341-1347, Sep. 1995.

[15] E. Altman and Z. Altman, "S-modular games and power control in wireless networks," IEEE Trans. Auto. Control vol. 48, no. 5, pp. 839842, 2003.

[16] T. Heikkinen, "A potential game approach to distributed power control and scheduling," Computer Networks vol. 50, pp. 2295-2311, 2006.

[17] R. Myerson, Game theory: Analysis of conflict, Cambridge and London: Harvard University Press, 1991.

[18] D. Monderer and L. S. Shapley, "Potential games," Games and Economics Behaviour, vol. 14, pp. 124-143, 1996. 